\title{
Separation and Analysis of Chlorpromazine and Demethylchlorpromazineby Capillary Electrophoresis Coupled with Electrochemiluminescence
}

\author{
Fuxiu Yang ${ }^{1}$, Zhaokuai Peng ${ }^{1,2}$, Chunxiu Gu ${ }^{1,2}$,Baining Liu ${ }^{1,2}$, Kaowen Zhou ${ }^{1,2, *}$ \\ ${ }^{1}$ Biochemical Engineering College, Beijing Union University, Beijing 100023, China \\ ${ }^{2}$ Beijing Key Laboratory of Biomass Waste Resource Utilization, Beijing 100023, China \\ *E-mail: zhoukaowen@ buu.edu.cn
}

doi: $10.20964 / 2019.07 .48$

Received: 4 February 2019/ Accepted: 24 March 2019 / Published: 10 June 2019

\begin{abstract}
Demethylchlorpromazine (DMCPZ) is one of the main metabolites of chlorpromazine (CPZ). DMCPZ has only one methyl less than CPZ. The structural difference between them is too small to separate them by conventional capillary electrophoresis (CE).However, proper addition of additives in the separation buffer can improve their separation performance. The parameters related to CE separation were carefully studied. The optimized CE conditions were separation buffer of $17.50 \%$ iso-propyl alcohol aqueous solution (v/v) containing $21 \mathrm{mmol} / \mathrm{L}$ phosphate $(\mathrm{pH} 5.3)$, separation voltage of 13.1 $\mathrm{kV}$, sample injection time of $6 \mathrm{~s}$ and sample injection voltage of $11.5 \mathrm{kV}$. Combined with electrochemiluminescence (CEL) detection technology, a separation and analysis method of CPZ and DMCPZ by improved CE coupled with end-column CEL was established. The limits of detection $(3 \sigma)$ of this method were $8.1 \times 10^{-7} \mathrm{mg} / \mathrm{mL}$ for CPZ and $8.9 \times 10^{-6} \mathrm{mg} / \mathrm{mL}$ for DMCPZ. The relative standard deviations were less than $2.5 \%$ for ECL intensity and less than $1.2 \%$ for migration time. This method had many advantages, such as fast speed, high sensitivity, small sample injection, and free from interference, and was successfully applied to simultaneously determine CPZ and DMCPZ in whole urine sample of pet dog.
\end{abstract}

Keywords: Improved capillary electrophoresis; Electrochemiluminescence; Chlorpromazine; Demethylchlorpromazine; Urine sample

\section{$\underline{\text { FULL TEXT }}$}

(C) 2019 The Authors. Published by ESG (www.electrochemsci.org). This article is an open access article distributed under the terms and conditions of the Creative Commons Attribution license (http://creativecommons.org/licenses/by/4.0/). 